\title{
ON THE INVARIANT SYSTEM OF A PAIR OF CONNEXES*
}

BY

\author{
O. E. GLENN
}

The theoretical part of this paper treats of a transvectant operation between two general connexes

$$
\begin{aligned}
& \phi=A a_{1 x} a_{2 x} \cdots a_{r x} \alpha_{1 u} \alpha_{2 u} \cdots \alpha_{s u}, \\
& \psi=B b_{1 x} b_{2 x} \cdots b_{\rho x} \beta_{1 u} \beta_{2 u} \cdots \beta_{\sigma u},
\end{aligned}
$$

wherein the variables are ternary and $(u)$ contragredient to $(x)$. This operation, defined by polarization, gives all invariant formations of the simultaneous system of two connexes. Our transvectant, of four indices,

$$
\tau=(\phi, \psi)_{k, l}^{i, j},
$$

can be interpreted in terms of convolution operations employed by Gordan in his memoirs on the systems of the ternary cubic and quartic forms, $\dagger$ and in Maisano's paperł on the concomitants of the first five degrees of the ternary quartic. For doubly homogeneous quantics which are in normal form§ $(\S 1,(4))$ the Formenreihe employed by E. Noether $\|$ includes an ordered succession of terms of transvectants $\tau$. As compared with the methods of the latter author, who dealt with normal ground-forms and therefore with a canonical form of the problem, our algorithm is non-canonical, and therefore symmetrical. The present method bears a closer resemblance to that of Clebsch and Gordan $\uparrow$ than to subsequently developed methods.

However, as preliminary to the application of the four types of convolution which were employed by all of the before-mentioned authors, we apply to one of the forms $\phi, \psi$ to be combined, a single analytic operation of polarization, involving $i$-fold operative factors corresponding to the first type of

* Presented to the Society, February 27, 1915.

† Gordan, M a the matis che Annalen, vol. 1 (1869), p. 90, and vol. 17 (1880), p. 217.

$\ddagger$ Maisano, Giornali di B at taglini, vol. 19.

$\S$ Gordan, M a th e m a t is ch e A n n a l e n, vol. 5 (1873), p. 104, and Programmschrift (Leipsic, 1875).

॥ E. Noether, Ueber die Bildung des Formensystems der ternären biquadratischen Form, Journal für Mathematik, vol. 134 (1908).

II Ueber biternäre Formen mit contragredienten Variabeln, $\mathrm{M}$ a the $\mathrm{mat}$ is c he $\mathrm{An}$ n a l e n, vol. 1 (1869), p. 359. 
convolution, $j$-fold operators corresponding to the second type, and $k$ - and $l$-fold operators corresponding respectively to the third and fourth types of convolution. The substitutions (1) made in this polar give $\tau$. This secures an ordering of the convolutions corresponding to a given set of indices $i, j$, $k, l$, so that theorems analogous to those on the difference between two terms of a binary transvectant are readily stated and proved for $\tau$. The preliminary operation of polarization was not employed by Clebsch and Gordan, although they, and likewise E. Noether (loc. cit.), employed the four types of convolution (under different schemes of ordering) to generate the complete systems which they treated.*

Where it is possible to proceed by applying convolutions between two systems which are finite and complete, absolutely or with respect to a set of symbolical moduli, $\tau$ yields all invariant formations $(\$ 1)$. Thus if we are given the finite complete system of each connex $\phi, \psi$, then the system derived by transvection between these two systems is itself finite and complete.

In the second part of the paper we employ $\tau$ in deriving a complete system of simultaneous concomitants of a quadric $p_{x}^{2}$ and a bilinear connex $a_{x} \alpha_{u}(\S 2)$. The problem of making reductions within the finite system generated by transvection in this case is treated from the two standpoints of reduction by identities, and by the syzygies connecting the ground forms. It is the author's thought that the present methods present possibilities for extension towards a theory for the general orders when an adequate asyzygetic theory of ternary forms is available.

We give below a list of memoirs on the various known methods of constructing ternary systems. $\dagger$

\section{Transvectant systems}

Let $\phi, \psi$ be the two general connexes given above. We define the transvectant $\tau$, of four indices, as follows: Polarize $\phi$ by the following operator,

$$
\begin{gathered}
\sum\left(y_{1}^{(1)} \frac{\partial}{\partial x}\right)^{\epsilon_{1}}\left(y_{2}^{(1)} \frac{\partial}{\partial x}\right)^{\epsilon_{2}} \cdots\left(y_{\sigma}^{(1)} \frac{\partial}{\partial x}\right)^{\epsilon_{\sigma}}\left(y_{1}^{(2)} \frac{\partial}{\partial x}\right)^{\iota_{1}}\left(y_{2}^{(2)} \frac{\partial}{\partial x}\right)^{\iota_{2}} \cdots\left(y_{\rho}^{(2)} \frac{\partial}{\partial x}\right)^{\iota_{\rho}} \\
\times\left(v_{1}^{(1)} \frac{\partial}{\partial u}\right)^{\sigma_{1}}\left(v_{2}^{(1)} \frac{\partial}{\partial u}\right)^{\sigma_{2}} \cdots\left(v_{\rho}^{(1)} \frac{\partial}{\partial u}\right)^{\sigma_{\rho}}\left(v_{1}^{(2)} \frac{\partial}{\partial u}\right)^{\nu_{1}}\left(v_{2}^{(2)} \frac{\partial}{\partial u}\right)^{\nu_{2}} \cdots\left(v_{\sigma}^{(2)} \frac{\partial}{\partial u}\right)^{\nu_{\sigma}},
\end{gathered}
$$
where

$$
\left(y_{i}^{(j)} \frac{\partial}{\partial x}\right)=y_{1 i}^{(j)} \frac{\partial}{\partial x_{1}}+y_{2 i}^{(j)} \frac{\partial}{\partial x_{2}}+y_{3 i}^{(j)} \frac{\partial}{\partial x_{3}},
$$

* The introduction of normal ground-forms enabled E. Noether to reduce the number of processes of convolution to two essential types.

† Baker, Cambridge Philosophical Transactions, vol. 15 (1889). Forsyth, American Journal of Mathematics, vol. 12 (1890). White, American Journal of Mathematics, vol. 14 (1892). Mertens, Wiener B erichte, vol. 95 . 
etc., and $\epsilon_{i}, \iota_{i}, \sigma_{i}, \nu_{i}=0$ or 1 , and

$$
\begin{array}{rlll}
\sum \epsilon=i, & \sum l=j, & \sum \sigma=k, & \sum \nu=l, \\
i+j \leqq r, & k+l \leqq s, & i+l \leqq \sigma, & j+k \leqq \rho .
\end{array}
$$

Substitute in the resulting polar $(\phi)$

$$
\begin{array}{lll}
\text { (a) } y_{p}^{(1)}=\beta_{p} & (p=1, \cdots, \sigma), \\
\text { (b) } y_{p}^{(2)}=\left(b_{p} u\right) & (p=1, \cdots, \rho), \\
\text { (c) } v_{p}^{(1)}=b_{p} & (p=1, \cdots, \rho), \\
\text { (d) } v_{p}^{(2)}=\left(\beta_{p} x\right) & (p=1, \cdots, \sigma),
\end{array}
$$

and multiply each term of the result by the $b_{x}, \beta_{u}$ factors whose symbols are not then involved in it. The resulting concomitant we call the transvectant of $\phi$ and $\psi$ of index $\left(\begin{array}{l}i, j \\ k, l\end{array}\right)$ and abbreviate it as

$$
\tau=(\phi, \psi)_{k, l}^{i, j} .
$$

We abbreviate the above polar as

$$
(\phi)=\sum \Delta_{y^{(1)}} \Delta_{y^{(2)}} \Delta_{v^{(1)}} \Delta_{v^{(2)}} \phi .
$$

Let $t_{1}, t_{2}$ be any two terms of the double mixed polar $(\phi)$. These can written, respectively,

$$
t_{1}=\gamma_{1} U_{1}, \quad t_{2}=\gamma_{2} U_{2},
$$

where $\gamma_{i}(i=1,2)$ are the products of the $a_{x}, a_{y}$ factors of $t_{i}(i=1,2)$, and $U_{i}(i=1,2)$ are the products of the $\alpha_{u}, \alpha_{v}$ factors of $t_{i}(i=1,2)$, respectively. Leaving out of account the constant factor $A,(\phi)$ is an expression of the type

$$
(\phi)=\sum\left(\gamma_{1}+\gamma_{2}+\cdots\right)\left(U_{1}+U_{2}+\cdots\right)=t_{1}+t_{2}+\cdots,
$$

wherein

$$
\sum \gamma=\frac{\Delta_{y^{(1)} \Delta_{y^{(2)} \phi}}}{\alpha_{1 u} \cdots \alpha_{s u}}, \quad \sum U=\frac{\Delta_{v^{(1)} \Delta_{v^{(2)} \phi}}}{a_{1 x} \cdots a_{r x}} .
$$

The first theorem to be proved depends upon the difference $t_{1}-t_{2}$ of two terms of $(\phi)$. We have

$$
t_{1}-t_{2}=\gamma_{1}\left(U_{1}-U_{2}\right)+U_{2}\left(\gamma_{1}-\gamma_{2}\right),
$$

and in this each parenthesis is the difference between two terms of a simple mixed polar. Now two terms of a simple polar are said to be adjacent when they differ only in that a pair of factors $a_{h y} a_{j x}$ in one is replaced in the other by $a_{h x} a_{j y}$. By a known theorem we can add and subtract terms within each 
parenthesis so that we will have

$$
\begin{aligned}
\gamma_{1}-\gamma_{2} & =\left[\gamma_{1}-\eta^{\prime}\right]+\left[\eta^{\prime}-\eta^{\prime \prime}\right]+\cdots+\left[\eta^{(i)}-\gamma_{2}\right], \\
U_{1}-U_{2} & =\left[U_{1}-\xi^{\prime}\right]+\left[\xi^{\prime}-\xi^{\prime \prime}\right]+\cdots+\left[\xi^{(j)}-U_{2}\right],
\end{aligned}
$$

where each bracket is a difference between two adjacent terms of a simple mixed polar.

Definition. The sum of the order of a form in the variables $(x)$ plus its order in the variables $(u)$ is called its grade.

Definition. The process by which we obtain from $\phi=a_{1 x} \cdots a_{r x} \alpha_{1 u} \cdots \alpha_{s u}$ any one of the three covariant types

$$
\begin{aligned}
& c_{1}=\left(a_{1} a_{2} a_{3}\right) a_{4 x} \cdots a_{r x} \alpha_{1 u} \cdots \alpha_{s u}, \\
& c_{2}=\left(\alpha_{1} \alpha_{2} \alpha_{3}\right) a_{1 x} \cdots a_{r x} \alpha_{4 u} \cdots \alpha_{s u}, \\
& c_{3}=a_{1 a_{1}} a_{2 x} \cdots a_{r x} \alpha_{2 u} \cdots \alpha_{s u},
\end{aligned}
$$

is called convolution.

THEOREM 1. The difference between any two terms $t_{1}, t_{2}$ of a transvectant

$$
\tau=(\phi, \psi)_{k, l}^{i, j},
$$

equals a sum of terms each of which is a term of a transvectant $\bar{\tau}$ of two forms $\bar{\phi}$, $\bar{\psi}$, at least one of which is of lower grade than the corresponding one in $\tau$. Moreover, $\bar{\phi}, \bar{\psi}$ are obtained from $\phi, \psi$ respectively by convolution.

To prove this theorem observe that the differences involved in (2) are essentially of ten types, and except for factors which may be left out of account in pursuing the argument these are
(A) $\quad a_{h y_{p}^{(1)}} a_{k x}-a_{k y_{p}^{(1)}} a_{h x}$
(D) $\quad a_{h y_{p}^{(2)}} a_{k y_{q}^{(2)}}-a_{k y_{p}^{(2)}} a_{h y_{q}^{(2)}}$,
(B) $a_{h y_{p}^{(1)}} a_{k y_{q}^{(1)}}-a_{k y_{p}^{(1)}} a_{h y_{q}^{(1)}}$,
(E) $a_{h y_{p}^{(2)}} a_{k x}-a_{k y_{p}^{(2)}} a_{h x}$,
(C) $a_{h y_{p}^{(1)}} a_{k y_{q}^{(2)}}-a_{k y_{p}^{(1)}} a_{h y_{q}^{(2)}}$,
( $\Delta) \quad \alpha_{h v_{p}^{(2)}} \alpha_{k v_{q}^{(2)}}-\alpha_{k v_{p}^{(2)}} \alpha_{h v_{q}^{(2)}}$,
(A) $\alpha_{h v_{p}^{(1)}} \alpha_{k u}-\alpha_{k v_{p}^{(1)}} \alpha_{h u}$,
(B) $\alpha_{h v_{p}^{(1)}} \alpha_{k v_{q}^{(1)}}-\alpha_{k v_{p}^{(1)}} \alpha_{h v_{q}^{(1)}}$,
(E) $\alpha_{h v_{p}^{(2)}} \alpha_{k u}-\alpha_{k v_{p}^{(2)}} \alpha_{h u}$.
(Г) $\alpha_{h v_{p}^{(1)}} \alpha_{k v_{q}^{(2)}}-\alpha_{k v_{p}^{(1)}} \alpha_{h v_{q}^{(2)}}$,

Substitution from (1) in these differences gives all types of expressions which occur in the difference between two terms of the transvectant $\tau$. The results of the substitutions are given below, $(A)$ going into $\left(A_{1}\right),(B)$ into $\left(B_{1}\right)$, etc.

$$
\begin{aligned}
& \left(A_{1}\right) \quad a_{h \beta_{p}} a_{k x}-a_{k_{\beta_{p}}} a_{h x}=\left(a_{h} a_{k}\left(\beta_{p} x\right)\right), \\
& \left(B_{1}\right) \quad a_{h \beta_{p}} a_{k \beta_{q}}-a_{k_{\beta_{p}}} a_{h \beta_{q}}=\left(a_{h} a_{k}\left(\beta_{p} \beta_{q}\right)\right),
\end{aligned}
$$


$\left(C_{1}\right) \quad a_{h_{\beta_{p}}}\left(a_{k} b_{q} u\right)-a_{k_{\beta_{p}}}\left(a_{h} b_{q} u\right)=\left(a_{h} a_{k} b_{q}\right) \beta_{p u}-\left(a_{h} a_{k} u\right) b_{q \beta_{p}}$,

$\left(D_{1}\right) \quad\left(a_{h} b_{p} u\right)\left(a_{k} b_{q} u\right)-\left(a_{k} b_{p} u\right)\left(a_{h} b_{q} u\right)=\left(a_{h} a_{k} u\right)\left(b_{p} b_{q} u\right)$,

$\left(E_{1}\right) \quad\left(a_{h} b_{p} u\right) a_{k x}-\left(a_{k} b_{p} u\right) a_{h x}=\left(a_{h} a_{k} u\right) b_{p x}-\left(a_{h} a_{k} b_{p}\right) u_{x}$.

The five expressions $\left(A_{1}\right),\left(B_{1}\right),\left(\Gamma_{1}\right),\left(\Delta_{1}\right),\left(E_{1}\right)$ are obtainable from the respective expressions $\left(A_{1}\right),\left(B_{1}\right),\left(C_{1}\right),\left(D_{1}\right),\left(E_{1}\right)$ by the substitutions

$$
\left(\begin{array}{lllllll}
a_{h} & a_{k} & \beta_{p} & \beta_{q} & b_{p} & b_{q} & u \\
\alpha_{h} & \alpha_{k} & b_{p} & b_{q} & \beta_{p} & \beta_{q} & x
\end{array}\right) .
$$

The right-hand sides of these expressions represent terms of transvectants as follows; the particular convolution from $\phi$ in which the factor $a_{h x} a_{k x}$ is replaced by $\left(a_{h} a_{k} u\right)$ being indicated by $\left(a_{h} a_{k} u\right) \phi^{\prime}$, and so forth:

$\left(A_{1}\right) \quad\left(\left(a_{h} a_{k} u\right) \phi^{\prime}, \psi\right)_{k, l+1}^{i-1, j}$,

$\left(B_{1}\right) \quad\left(\left(a_{h} a_{k} u\right) \phi^{\prime},\left(\beta_{p} \beta_{q} x\right) \psi^{\prime}\right)_{k+1, l}^{i-2, j}$,

$\left(C_{1}\right) \quad\left(\left(a_{h} a_{k} u\right) \phi^{\prime}, \psi\right)_{k+1, l}^{i-1, j-1}$, and $\left(\left(a_{h} a_{k} u\right) \phi^{\prime}, b_{q \beta_{p}} \psi^{\prime}\right)_{k, l}^{i-1, j-1}$,

$\left(D_{1}\right) \quad\left(\left(a_{h} a_{k} u\right) \phi^{\prime},\left(b_{p} b_{q} u\right) \psi^{\prime}\right)_{k, l}^{i, j-2}$,

$\left(E_{1}\right) \quad\left(\left(a_{h} a_{k} u\right) \phi^{\prime}, \psi\right)_{k, l}^{i, j-1}$, and $\left(\left(a_{h} a_{k} u\right) \phi^{\prime}, \psi\right)_{k+1, l}^{i, j-1} u_{x}$.

Inasmuch as $\left(A_{1}\right), \cdots,\left(E_{1}\right)$ arise from $\left(A_{1}\right), \cdots,\left(E_{1}\right)$ by $(3)$, the principle of symmetry in the theory now immediately shows that the theorem is true in all cases, since $\left(a_{h} a_{k} u\right) \phi^{\prime}$ is of lower grade than $\phi$ and $\left(\alpha_{h} \alpha_{k} x\right) \psi^{\prime}$ is of lower grade than $\psi$.

An immediate corollary is that any monomial concomitant $\kappa$ equals the transvectant $\tau$ of which it is a term plus terms of transvectants of forms one at least of which is a convolution from the corresponding one in $\tau$ and hence of lower grade, times powers of $u_{x}$.

Evidently a successive application of this corollary gives for $\kappa$ an expansion in the form

$$
\kappa=w_{0}+w_{1} u_{x}+w_{2} u_{x}^{2}+\cdots .
$$

Gordan and also Mertens derived a similar result and showed that the $w_{i}$ were normal forms, i. e. they satisfy the equation

$$
\frac{\partial^{2} w}{\partial x_{1} \partial u_{1}}+\frac{\partial^{2} w}{\partial x_{2} \partial u_{2}}+\frac{\partial^{2} w}{\partial x_{3} \partial u_{3}}=0 \text {. }
$$

Theorem 2. Let $\phi=A_{x}^{r} B_{u}^{s}, \psi=C_{x}^{\rho} D_{u}^{\sigma}$, and let $\tau$ be the transvectant

$$
\tau=(\phi, \psi)_{k, l}^{i, j} \text {. }
$$

Then if it be possible to separate $\phi, \psi$ into factors,

$$
\begin{aligned}
& \phi=\phi_{1} \phi_{2}=A_{1 x}^{r_{1}} B_{1 u}^{s_{1}} \cdot A_{2 x}^{r_{2}} B_{2 u}^{s_{2}}, \\
& \psi=\psi_{1} \psi_{2}=C_{1 x}^{\rho_{1}} D_{1 u}^{\sigma_{1}} \cdot C_{2 x}^{\rho_{2}} D_{2 u}^{\sigma_{2}},
\end{aligned}
$$


and to partition the index numbers,

$$
i=i_{1}+i_{2}, \quad j=j_{1}+j_{2}, \quad k=k_{1}+k_{2}, \quad l=l_{1}+l_{2},
$$

in such a way that the transvectants

$$
\tau_{1}=\left(\phi_{1}, \psi_{1}\right)_{k_{1}, l_{1}}^{i_{1}, j_{1}}, \quad \tau_{2}=\left(\phi_{2}, \psi_{2}\right)_{k_{2}, l_{2}}^{i_{2}, j_{2}}
$$

exist as separate nonsymbolical entities, then $\tau$ contains reducible terms, namely, terms of the product $\tau_{1} \tau_{2}$.

The transvectant $\tau$ is evaluated by polarizing $\phi$ by means of the operator

and substituting

$$
\left(y^{(1)} \frac{\partial}{\partial x}\right)^{i}\left(y^{(2)} \frac{\partial}{\partial x}\right)^{j}\left(v^{(1)} \frac{\partial}{\partial u}\right)^{k}\left(v^{(2)} \frac{\partial}{\partial u}\right)^{l},
$$

$$
y^{(1)}=D, \quad y^{(2)}=(C u), \quad v^{(1)}=C, \quad v^{(2)}=(D x) .
$$

Thus $\tau$ contains the term

$$
\begin{gathered}
P\left(y_{1}^{(1)} \frac{\partial}{\partial x}\right)^{i_{1}}\left(y_{1}^{(2)} \frac{\partial}{\partial x}\right)^{j_{1}} A_{1 x}^{r_{1}}\left(y_{2}^{(1)} \frac{\partial}{\partial x}\right)^{i_{2}}\left(y_{2}^{(2)} \frac{\partial}{\partial x}\right)^{j_{2}} A_{2 x}^{r_{2}} \\
\times\left(v_{1}^{(1)} \frac{\partial}{\partial u}\right)^{k_{1}}\left(v_{1}^{(2)} \frac{\partial}{\partial u}\right)^{l_{1}} B_{1 u}^{s_{1}}\left(v_{2}^{(1)} \frac{\partial}{\partial u}\right)^{k_{2}}\left(v_{2}^{(2)} \frac{\partial}{\partial u}\right)^{l_{2}} B_{2 u}^{s_{3}} \\
{\left[y_{1}^{(1)}=D_{1}, \quad y_{1}^{(2)}=\left(C_{1} u\right), \quad y_{2}^{(1)}=D_{2}, \quad y_{2}^{(2)}=\left(C_{2} u\right),\right.} \\
\left.v_{1}^{(1)}=C_{1}, \quad v_{1}^{(2)}=\left(D_{1} x\right), \quad v_{2}^{(1)}=C_{2}, \quad v_{2}^{(2)}=\left(D_{2} x\right)\right] .
\end{gathered}
$$

This term is a constant times

$$
\tau_{1} \tau_{2}=\left(\phi_{1}, \psi_{1}\right)_{k_{1}, l_{1}}^{i_{1}, j_{1}}\left(\phi_{2}, \psi_{2}\right)_{k_{2}, l_{2}}^{i_{2}, j_{2}} .
$$

Let $[\phi]$ represent a system of connexes given explicitly by

$$
\phi_{i}=a_{i x}^{\omega_{i}} \alpha_{i u}^{\mu_{i}} \quad(i=1,2, \cdots),
$$

and let $[\psi]$ represent the system

$$
\psi_{i}=b_{i x}^{\eta_{i}} \beta_{i u}^{\gamma_{i}} \quad(i=1,2, \cdots) .
$$

Then a system $[\tau]$ is said to be the system derived from $[\phi],[\psi]$ by transvection when it includes all terms of all transvectants,

$$
\tau=(U, V)_{k, l}^{i, j},
$$

where $U$ is a product of powers of forms from the system [ $\phi]$ and $V$ a product of powers of forms of the system $[\psi]$.

Theorem 3. The number of transvectants of the system [ $\tau]$ which contain no reducible terms is finite.*

* Gordan proved the finiteness of simultaneous systems in 1875. 
In proof let

$$
U=\phi_{1}^{p_{1}} \phi_{2}^{p_{2}} \cdots \phi_{a}^{p_{a}}, \quad V=\psi_{1}^{q_{1}} \psi_{2}^{q_{2}} \cdot \psi_{b}^{q_{b}} .
$$

Then the order of $U$ in the $p=p_{1}+\cdots+p_{a}$ distinct $a$ symbols is

$$
p_{1} \omega_{1}+p_{2} \omega_{2}+\cdots+p_{a} \omega_{a},
$$

and the order of $U$ in the $p$ distinct $\alpha$ symbols is

$$
p_{1} \mu_{1}+p_{2} \mu_{2}+\cdots+p_{a} \mu_{a} .
$$

The total effect of the transvection $\tau$ upon $U, V$ is to produce a sequence of terms by folding $i \beta$ symbols and $j b$ symbols into convolution combinations with $a$ symbols, and by folding $k b$ symbols and $l \beta$ symbols into convolution combinations with $\alpha$ symbols. In one of these terms $t_{1}$ of $\tau$ let the number of $a$ symbols not in convolution combination with other symbols be $\rho_{1}$. Likewise let the number of $\alpha$ symbols of $t_{1}$ not so combined be $\rho_{2}$, the number of $b$ symbols not combined be $\rho_{3}$, and the number of $\beta$ symbols not combined with other symbols be $\rho_{4}$. Then we have

$$
\begin{aligned}
p_{1} \omega_{1}+p_{2} \omega_{2}+\cdots+p_{a} \omega_{a} & =\rho_{1}+i+j, \\
p_{1} \mu_{1}+p_{2} \mu_{2}+\cdots+p_{a} \mu_{a} & =\rho_{2}+k+l, \\
q_{1} \eta_{1}+q_{2} \eta_{2}+\cdots+q_{b} \eta_{b} & =\rho_{3}+j+k, \\
q_{1} \gamma_{1}+q_{2} \gamma_{2}+\cdots+q_{b} \gamma_{b} & =\rho_{4}+i+l .
\end{aligned}
$$

We thus have a system of four linear diophantine equations in the variables $p_{1}, \cdots, p_{a}, q_{1}, \cdots, q_{b}, \rho_{1}, \cdots, \rho_{4}, i, j, k, l$, to be satisfied in positive integers. Each reducible solution of this system corresponds to a transvectant $\tau$ having reducible terms, by the theorem last proved. But the number of irreducible solutions of such a system is finite by a well-known theorem. Hence the theorem is proved.

Definition. A system of connexes containing an infinite number of individuals is said to be finite when there exists a finite set of the connexes such that every connex of the system is a rational integral function of the members of the set.

Definition. A system is complete when any form obtained from a product of connexes of the system, by convolution, is expressible rationally and integrally in terms of connexes of the system.

A proof of a theorem now follows readily from the properties of transvectants established above, giving a method of constructing the finite system of a pair of connexes.

TheOREM 4. If two infinite systems of connexes $[\phi],[\psi]$ are each finite and complete then the system $[\tau]$ derived therefrom by transvection is finite and complete. 
To prove the finiteness we have only to arrange the transvectants of $[\tau]$ in an ordered sequence arranged according to ascending degrees, those of the same degree being ordered according to ascending grade of the product $U V$, where the typical transvectant of the sequence is

$$
\tau=(U, V)_{k, i}^{i, j} \text {. }
$$

Since the difference between any two terms of $\tau$ is a sum of terms of transvectants,

$$
\bar{\tau}=(\bar{U}, \bar{V}) \underset{k^{\prime}, i^{\prime},}{i, j^{\prime},}
$$

where $\bar{U} \bar{V}$ is of lower grade than $U V$, a set of connexes in terms of which all others of $[\tau]$ may be rationally expressed may be obtained by selecting terms of the transvectants in this sequence, and precisely one term from each transvectant which contains no reducible term. But the number of transvectants in $[\tau]$ which contain no reducible terms is finite.

To prove completeness let the finite set described above be $K_{1}, \cdots, K_{t}$, and let $\bar{P}$ be any monomial form obtained by convolution from

$$
P=K_{1}^{k_{1}} K_{2}^{k_{2}} \cdots K_{t}^{k_{t}} .
$$

Then $\bar{P}$ is a term of a transvectant

$$
(\bar{U}, \bar{V})_{k, \imath}^{i, \jmath} u_{x}^{e}
$$

and in which $\bar{U}$ is obtainable by convolution from [ $\phi]$ forms alone, and $\bar{V}$ from $[\psi]$ forms alone. Hence

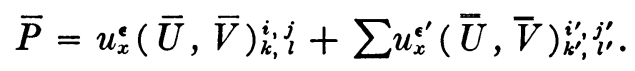

But $[\phi],[\psi]$ are, by assumption, complete systems, and consequently all transvectants on the right belong to the system $[\tau]$. Hence $[\tau]$ is complete.

\section{Fundamental system of a CONIC ANd a BILINEAR CONNEX}

We let $F=p_{x}^{2}=q_{x}^{2}=\cdots$ be a quadric, $L=(p q u)^{2}$ its contravariant, $D=(p q r)^{2}$ its discriminant . The connex $f=a_{x} \alpha_{u}=b_{x} \beta_{u}=\cdots$ has the fundamental system*

$$
\begin{gathered}
f, f_{1}=a_{x} \beta_{u} b_{\alpha}, \quad g=a_{x} c_{x}(\beta \gamma x) b_{\alpha}, \quad h=\beta_{u} \gamma_{u}(a c u) b_{\alpha}, \\
i=a_{a}, \quad i_{1}=a_{\beta} b_{a}, \quad i_{2}=a_{\beta} b_{\gamma} c_{a} .
\end{gathered}
$$

The simultaneous system of $F$ and $f$ is the transvectant system given by

$$
\left(F^{r} L^{s}, f^{t} f_{1}^{u} g^{v} h^{w}\right)_{k, l}^{i, j},
$$

together with the invariants $i, i_{1}, i_{2}, D$. There are, in addition to the

* Clebsch and Gordan, M a the m a ti s ch e A n n a le n, vol. 1 (1869), p. 359. 
members of the two individual systems, precisely 92 transvectants of this formula not immediately reducible by theorem (2) of $\S 1$. This is exclusive of the large number which are reducible by the known syzygy

$$
\begin{aligned}
& g h=\left|\begin{array}{ccc}
i^{\prime \prime} f-i i^{\prime \prime} u_{x}+i^{\prime} f_{2} & i^{\prime \prime} u_{x} & f_{2} \\
i^{\prime \prime} u_{x} & i f-i^{\prime} u_{x}+f_{2} & f \\
f_{2} & f & u_{x}
\end{array}\right| ; \\
& i^{\prime}=\frac{1}{2}\left(i^{2}-i_{1}\right), \quad i^{\prime \prime}=\frac{1}{6}\left(i^{3}-3 i i_{1}+2 i_{2}\right), \quad f_{2}=f_{1}-i f+i^{\prime} u_{x} .
\end{aligned}
$$

Of the 92 , twenty-three are reducible by the identity

$$
(a b u)(\rho \sigma x)=\left|\begin{array}{lll}
a_{\rho} & a_{\sigma} & a_{x} \\
b_{\rho} & b_{\sigma} & b_{x} \\
\rho_{u} & \sigma_{u} & u_{x}
\end{array}\right|,
$$

which is applicable in most cases where $l>0$. The remaining 69 forms are arranged in the following table according to ascending grade of $U V$, in accordance with the theory.

Table

Grade 4

$$
\begin{array}{rlrl}
(F, f)_{00}^{10} & =p_{a} p_{x} a_{x}, & & (F, f)_{00}^{01}=(p a u) \alpha_{u} p_{x}, \\
(F, f)_{00}^{11} & =(p a u) p_{a}, & & \left(F, f_{1}\right)_{00}^{10}=p_{\beta} b_{a} p_{x} a_{x}, \\
\left(F, f_{1}\right)_{00}^{01} & =b_{a}(p a u) \beta_{u} p_{x}, & & \left(F, f_{1}\right)_{00}^{11}=p_{\beta} b_{\alpha}(p a u), \\
(L, f)_{10}^{00} & =(p q a)(p q u) \alpha_{u}, & & (L, f)_{01}^{00}=(p q u)(\overline{p q} \alpha x) a_{x}, \\
(L, f)_{11}^{00}=(p q a)(\overline{p q} \alpha x), & & \left(L, f_{1}\right)_{10}^{00}=b_{a}(p q a)(p q u) \beta_{u}, \\
\left(L, f_{1}\right)_{01}^{00}=b_{a}(p q u)(\overline{p q} \beta x) a_{x}, & & \left(L, f_{1}\right)_{11}^{00}=b_{a}(p q a)(\overline{p q} \beta x) .
\end{array}
$$

Grade 5

$$
\begin{array}{lll}
(F, h)_{00}^{10}=b_{a} p_{\beta}(a c u) \gamma_{u} p_{x}, & (F, h)_{00}^{20}=b_{\alpha} p_{\beta} p_{\gamma}(a c u), \\
(L, g)_{10}^{00}=b_{\alpha}(p q a)(p q u)(\beta \gamma x) c_{x}, & (L, g)_{20}^{00}=b_{\alpha}(p q a)(p q c)(\beta \gamma x) .
\end{array}
$$

Grade 6

$$
\begin{array}{rlrl}
(F L, f)_{10}^{10} & =p_{a}(q r a)(q r u) p_{x}, & & \left(F L, f_{1}\right)_{10}^{10}=b_{a} p_{\beta}(q r a)(q r u) p_{x}, \\
\left(F, f^{2}\right)_{00}^{20} & =p_{a} p_{\beta} a_{x} b_{x}, & & \left(F, f^{2}\right)_{00}^{02}=(p a u)(p b u) \alpha_{u} \beta_{u}, \\
\left(F, f_{1}^{2}\right)_{00}^{20} & =b_{a} p_{\beta} p_{\delta} d_{\gamma} a_{x} c_{x}, & & \left(F, f_{1}^{2}\right)_{00}^{02}=b_{a} d_{\gamma}(p a u)(p c u) \beta_{u} \delta_{u}, \\
\left(F, f f_{1}\right)_{00}^{20}=b_{a} p_{\beta} p_{\gamma} a_{x} c_{x}, & \left(F, f f_{1}\right)_{00}^{02}=b_{a}(p a u)(p c u) \beta_{u} \gamma_{u}, \\
\left(L, f^{2}\right)_{20}^{00}=(p q a)(p q b) \alpha_{u} \beta_{u}, & \left(L, f^{2}\right)_{02}^{00}=(\overline{p q} \alpha x)(\overline{p q} \beta x) a_{x} b_{x}, \\
\left(L, f_{1}^{2}\right)_{20}^{00}=b_{a} d_{\gamma}(p q a)(p q c) \beta_{u} \delta_{u}, & \left(L, f_{1}^{2}\right)_{02}^{00}=b_{a} d_{\gamma}(\overline{p q} \beta x)(\overline{p q} \delta x) a_{x} c_{x}, \\
\left(L, f f_{1}\right)_{20}^{00}=b_{a}(p q a)(p q c) \beta_{u} \gamma_{u}, & \left(L, f f_{1}\right)_{02}^{00}=b_{a}(\overline{p q} \beta x)(\overline{p q} \gamma x) a_{x} c_{x} .
\end{array}
$$


Grade 7

$$
\begin{aligned}
& \left(F^{2}, g\right)_{00}^{03}=b_{a}(p a u)(p c u)(q \overline{\beta \gamma} u) q_{x}, \\
& \left(F^{2}, h\right)_{00}^{30}=b_{a} p_{\beta} p_{\gamma}(q a c) q_{x}, \bar{c} \\
& \left(L^{2}, g\right)_{30}^{00}=b_{a}(p q a)(p q c)(r s \overline{\beta \gamma})(r s u), \\
& \left(L^{2}, h\right)_{03}^{00}=b_{a}(\overline{p q} \beta x)(\overline{p q} \gamma x)(\overline{r s} \overline{a c} x)(r s u), \\
& (L F, g)_{20}^{01}=b_{a}(q r c)(q r \overline{\beta \gamma})(p a u) p_{x}, \\
& (F L, h)_{01}^{20}=b_{\alpha} p_{\beta} p_{\gamma}(q r u)(\overline{q r a c} x) .
\end{aligned}
$$

Grade 8

$$
\begin{aligned}
& \left(F L, f^{2}\right)_{10}^{20}=p_{a} p_{\beta}(q r a)(q r u) b_{x}, \\
& \left(F L, f^{2}\right)_{20}^{20}=p_{a} p_{\beta}(q r a)(q r b), \\
& \left(F L, f^{2}\right)_{20}^{10}=p_{a}(q r a)(q r b) p_{x} \beta_{u}, \\
& \left(F L, f_{1}^{2}\right)_{10}^{20}=b_{a} p_{\beta} p_{\delta} d_{\gamma}(q r a)(q r u) c_{x}, \\
& \left(F L, f_{1}^{2}\right)_{20}^{20}=b_{\alpha} p_{\beta} p_{\delta} d_{\gamma}(q r a)(q r c), \\
& \left(F L, f_{1}^{2}\right)_{20}^{10}=b_{\alpha} p_{\beta} d_{\gamma}(q r a)(q r c) \delta_{u} p_{x}, \\
& \left(F L, f f_{1}\right)_{10}^{20}=b_{a} p_{\beta} p_{\gamma}(q r a)(q r u) c_{x}, \\
& \left(F L, f f_{1}\right)_{20}^{20}=b_{\alpha} p_{\beta} p_{\gamma}(q r a)(q r c), \\
& \left(F L, f f_{1}\right)_{20}^{10}=b_{\alpha} p_{\beta}(q r a)(q r c) \gamma_{u} p_{x} .
\end{aligned}
$$

\section{Grade 9}

$$
\begin{aligned}
& \left(F^{2}, f g\right)_{n_{0}}^{04}=b_{a}(p a u)(p c u)(q d u)(q \overline{\beta \gamma} u) \delta_{u}, \\
& \left(F^{2}, f h\right)_{00}^{40}=b_{a} p_{\beta} p_{\gamma} q_{\delta}(q a c) d_{x} \text {, } \\
& \left(F^{2}, f_{1} g\right)_{00}^{04}=b_{a} e_{\delta}^{\prime}(p a u)(p c u)(q d u)(q \overline{\beta \gamma} u) \epsilon_{u} \text {, } \\
& \left(F^{2}, f_{1} h\right)_{00}^{\mathbf{4 0}}=b_{\alpha} e_{\delta} p_{\beta} p_{\gamma} q_{\mathrm{e}}(q a c) d_{x} \text {, } \\
& \left(L^{2}, f g\right)_{40}^{00}=b_{a}(p q a)(p q c)(r s d)(r s \overline{\beta \gamma}) \delta_{u} \text {, } \\
& \left(L^{2}, f h\right)_{04}^{00}=b_{\alpha}(\overline{p q} \beta x)(\overline{p q} \gamma x)(\overline{r s} \delta x)(\overline{r s} \overline{a c} x) d_{x} \text {, } \\
& \left(L^{2}, f_{1} g\right)_{40}^{00}=b_{\alpha} e_{\delta}(p q a)(p q c)(r s d)(r s \overline{\beta \gamma}) \epsilon_{u} \text {, } \\
& \left(L^{2}, f_{1} h\right)_{04}^{00}=b_{a} e_{\delta}(\overline{p q} \beta x)(\overline{p q} \gamma x)(\overline{r s \epsilon x})(\overline{r s} \overline{a c} x) d_{x} \text {, } \\
& (F L, f g)_{20}^{02}=b_{a}(q r d)(q r \overline{\beta \gamma})(p a u)(p c u) \delta_{u} \text {, } \\
& (F L, f h)_{02}^{20}=b_{\alpha} p_{\beta} p_{\gamma}(\overline{q r} \delta x)(\overline{q r} \overline{a c} x) d_{x} \text {, } \\
& \left(F L, f_{1} g\right)_{20}^{02}=b_{\alpha} e_{\delta}(q r d)(q r \overline{\beta \gamma})(p a u)(p c u) \epsilon_{u} \text {, } \\
& \left(F L, f_{1} h\right)_{02}^{20}=b_{\alpha} e_{\delta} p_{\beta} p_{\gamma}(\overline{q r} \in x)(\overline{q r} \overline{q c} x) d_{x} \text {. }
\end{aligned}
$$

Grade 10: No irreducible forms

\section{Grade 11}

$$
\begin{aligned}
& \left(F^{2} L, f h\right)_{10}^{40}=b_{a} p_{\beta} p_{\gamma} q_{\delta}(q a c)(r s d)(r s u) \\
& \left(F L^{2}, f g\right)_{40}^{10}=b_{a}(q r a)(q r c)(s t d)(s t \overline{\beta \gamma}) p_{\delta} p_{x}, \\
& \left(F^{2} L, f_{1} h\right)_{10}^{40}=b_{a} e_{\delta} p_{\beta} p_{\gamma} q_{\mathrm{e}}(q a c)(r s d)(r s u) \\
& \left(F L^{2}, f_{1} g\right)_{40}^{10}=b_{a} e_{\delta}(q r a)(q r c)(s t d)(s t \overline{\beta \gamma}) p_{c} p_{x}
\end{aligned}
$$


Grade 12

$$
\begin{aligned}
&\left(F^{3}, g^{2}\right)_{00}^{06}=b_{a} e_{\delta}(p a u)(p c u)(q d u)(q f u)(r \overline{\beta \gamma} u)(r \overline{\epsilon \eta} u), \\
&\left(L^{3}, g^{2}\right)_{60}^{00}=b_{a} e_{\delta}(o p a)(o p c)(q r d)(q r f)(\overline{s t} \beta \gamma)(\overline{s t} \epsilon \eta), \\
&\left(F^{3}, h^{2}\right)_{00}^{60}=b_{a} e_{\delta} p_{\beta} p_{\gamma} q_{e} q_{\eta}(r a c)(r d f), \\
&\left(L^{3}, h^{2}\right)_{06}^{00}=b_{a} e_{\delta}(\overline{o p} \beta x)(\overline{o p} \gamma x)(\overline{q r} \epsilon x)(\overline{q r} \eta x)(\overline{s t} \bar{c} x)(\overline{s t} \overline{d f} x), \\
&\left(F^{2} L, g^{2}\right)_{20}^{04}=b_{a} e_{\delta}(r s \overline{\beta \gamma})(r s \overline{\epsilon \eta})(\underline{p a u})(p c u)(q d u)(q f u), \\
&\left(F L^{2}, g^{2}\right)_{40}^{02}=b_{\alpha} e_{\delta}(q r d)(q r f)(s t \overline{\beta \gamma})(s t \overline{\epsilon \eta})(p a u)(p c u), \\
&\left(F^{2} L, h^{2}\right)_{02}^{40}=b_{a} e_{\delta} p_{\beta} p_{\gamma} q_{e} q_{\eta}(\overline{r s} \overline{a c} x)(\overline{r s} \overline{d f} x), \\
&\left(F L^{2}, h^{2}\right)_{04}^{20}=b_{a} e_{\delta} p_{\beta} p_{\gamma}(\overline{q r} \epsilon x)(\overline{q r} \eta x)(\overline{s t} \overline{a c} x)(\overline{s t} \overline{d f} x) .
\end{aligned}
$$

In order to illustrate the process of reduction of the transvectants which are reducible by (6) but not by theorem (2), $\S 1$, let us consider

This has the term

$$
\tau=(F L, h)_{01}^{10} \text {. }
$$

and

$$
t=b_{a} p_{\beta}(a c u)(q r u)(\overline{q r} \gamma x) p_{x}
$$

$$
t=b_{\alpha} p_{\beta}(q r u) p_{x}\left|\begin{array}{ccc}
(q r a) & a_{\gamma} & a_{x} \\
(q r c) & c_{\gamma} & c_{x} \\
(q r u) & \gamma_{u} & u_{x}
\end{array}\right| .
$$

We treat each term of this $t$ separately. Thus we deduce

$$
\begin{gathered}
(q r a)(q r u) b_{a} p_{\beta} p_{x} c_{\gamma} u_{x}=i \cdot u_{x} \cdot\left(F L, f_{1}\right)_{10}^{10}, \\
(q r c)(q r u) \gamma_{u} b_{\alpha} p_{\beta} p_{x} a_{x}=(L, f)_{10}^{00} \cdot\left(F, f_{1}\right)_{00}^{10}, \\
(q r u)^{2} b_{\alpha} a_{\gamma} p_{\beta} p_{x} c_{x}=L \cdot\left(F, a_{\gamma} \beta_{u} c_{x} b_{a}\right)_{00}^{10} .
\end{gathered}
$$

In the latter case we have*

$$
a_{\gamma} \beta_{u} b_{a} c_{x}=\frac{1}{6} u_{x}\left(i^{3}+2 i_{2}-3 i i_{1}\right)-\frac{1}{2}\left(i^{2} f-i_{1} f-2 i f_{1}\right) .
$$

Hence $\left(F, a_{\gamma} \beta_{u} c_{x} b_{\alpha}\right)_{00}^{10}$ is reducible in terms of invariants and

Furthermore,

$$
(F, f)_{00}^{10}, \quad\left(F, f_{1}\right)_{00}^{10}, \quad F \text {. }
$$

$$
\begin{gathered}
(q r u)^{2} c_{\gamma} b_{\alpha} p_{\beta} p_{x} a_{x}=i \cdot L \cdot\left(F, f_{1}\right)_{00}^{10}, \\
(q r a)(q r u) b_{a} p_{\beta} p_{x} \gamma_{u} c_{x}=f \cdot\left(F L, f_{1}\right)_{10}^{10}, \\
(q r c)(q r u) b_{\alpha} a_{\gamma} p_{\beta} p_{x} u_{x}=u_{x} \cdot\left(F L, a_{\gamma} \beta_{u} c_{x} b_{a}\right)_{10}^{10} .
\end{gathered}
$$

The latter form is reducible in terms of $u_{x},(F L, f)_{10}^{10},\left(F L, f_{1}\right)_{10}^{10}$, and invariants.

We have now proved that the term $t$ of $\tau$ is reducible.

\footnotetext{
* Clebsch and Gordan, loc. cit., p. 374.
} 
A different procedure is required in the case of transvectants of the type of

This form $\tau$ has the term

$$
\tau=(L, h)_{02}^{00} \text {. }
$$

anc $t$ equals

$$
t=(a c u)(\overline{p q} \beta x)(\overline{p q} \gamma x) b_{a},
$$

$$
b_{\alpha}(\overline{p q} \gamma x)\left|\begin{array}{lll}
(p q a) & a_{\beta} & a_{x} \\
(p q c) & c_{\beta} & c_{x} \\
(p q u) & \beta_{u} & u_{x}
\end{array}\right| .
$$

Expanding the latter determinant we have for one of $t$ 's terms

$$
t_{1}=(p q a)(\overline{p q} \gamma x) \beta_{u} c_{x} b_{a},
$$

and $t_{1}$ is a term of the transvectant

$$
\tau_{1}=\left(L, f f_{1}\right)_{11}^{00} .
$$

Another term of $\tau_{1}$ is the reducible form

$$
t_{2}=(p q a)(\overline{p q} \beta x) b_{\alpha} \gamma_{u} c_{x}=f \cdot\left(L, f_{1}\right)_{11}^{00},
$$

and we have, in accordance with the theory of $\S 1$,

$$
\begin{aligned}
t_{1}-t_{2} & =(p q a) b_{a} c_{x}\left[(\overline{p q} \gamma x) \beta_{u}-(\overline{p q} \beta x) \gamma_{u}\right] \\
& =(p q a)(p q u)(\beta \gamma x) c_{x} b_{a}-(p q a)(p q \overline{\beta \gamma}) c_{x} b_{\alpha} u_{x} .
\end{aligned}
$$

The terms of this last difference are terms of the respective transvectants

$$
(L, g)_{10}^{00}, \quad(L, g)_{20}^{00} \cdot u_{x},
$$

and these are retained in the above table. Thus $t_{1}$ is reducible, in the form

$$
t_{1}=f \cdot\left(L, f_{1}\right)_{11}^{00}+(L, g)_{10}^{00}-u_{x} \cdot(L, g)_{20}^{00}+\Sigma \text {, }
$$

where $\Sigma$ is a sum of terms of transvectants $u_{x}^{e}(L, \bar{g})_{k l}^{i j}, \bar{g}$ being derived by convolution from $g$ and hence of lower grade than $g$ and thus linear in $f, f_{1}$, $u_{x}$ and invariants. The other terms of the expanded $t$, with minus signs changed, are

$$
\begin{aligned}
(p q a)(\overline{p q} \gamma x) b_{\alpha} c_{\beta} u_{x} & =\left(L, a_{x} b_{\alpha} c_{\beta} \gamma_{u}\right)_{11}^{00} u_{x} \\
& =\left(L, a_{\gamma} \beta_{u} c_{x} b_{a}\right)_{11}^{00} u_{x}, \\
(p q c)(\overline{p q} \gamma x) a_{x} \beta_{u} b_{a} & =f_{1}(L, f)_{11}^{00}, \\
(p q u)(\overline{p q} \gamma x) a_{\beta} b_{\alpha} c_{x} & =i_{1}(L, f)_{01}^{00}, \\
(p q u)(\overline{p q} \gamma x) a_{x} c_{\beta} b_{a} & =\left(L, a_{x} b_{\alpha} c_{\beta} \gamma_{u}\right)_{01}^{00}, \\
(p q c)(\overline{p q} \gamma x) a_{\beta} b_{a} u_{x} & =i_{1} \cdot u_{x} \cdot(L, f)_{11}^{00}
\end{aligned}
$$


Hence the term $t$ of $\tau$ is reducible in terms of forms of lower degree, together with forms which are retained in the table and forms which are terms of transvectants for which the grade of $U V$ is less than the grade of $L h$. Hence $\tau=(L, h)_{02}^{00}$ does not belong to the fundamental system.

The remaining 21 reducible transvectants are as follows:
$\left(F L, f^{2}\right)_{02}^{02}$,
$\left(F L, f_{1}^{2}\right)_{02}^{02}$
$\left(F L, f f_{1}\right)_{02}^{02}$
$(F L, f)_{01}^{01}$
$\left(F L, f^{2}\right)_{01}^{02}$,
$\left(F L, f^{2}\right)_{02}^{01}$,
$\left(F L, f_{1}\right)_{01}^{01}$,
$\left(F L, f_{1}^{2}\right)_{01}^{02}$,
$\left(F L, f_{1}^{2}\right)_{02}^{01}$,
$\left(F L, f f_{1}\right)_{01}^{02}$,
$\left(F L, f f_{1}\right)_{02}^{01}$
$(F, g)_{00}^{01}$,
$(L, h)_{01}^{00}$,
$(F L, g)_{10}^{01}$,
$(F, g)_{00}^{02}$,
$(F L, g)_{10}^{02}$
$(F L, h)_{02}^{10}$,
$\left(F^{2} L, f g\right)_{01}^{04}, \quad\left(F L^{2}, f h\right)_{04}^{01}, \quad\left(F^{2} L, f_{1} g\right)_{01}^{04}$ ，
$\left(F L^{2}, f_{1} h\right)_{04}^{01}$.
University of Pennsylvania 